Supporting Information for

\title{
Ultra-strong anion affinity of anionic clay induced by its inherent
}

\section{nanoconfinement}

Chen Tian ${ }^{\dagger, \ddagger}$, Jingwei Tu ${ }^{\dagger, \S}$, Peipeng Qiu ${ }^{\dagger, \ddagger}$, Shuting Wang ${ }^{\dagger, \star}$, Han Song ${ }^{\dagger, \sharp}$, Yunyun $\mathrm{Xu}^{\dagger, \star}$, Xu Yan", Zhang Lin ${ }^{\dagger,}$, ",, , Liyuan Chai"

$\dagger$ School of Environment and Energy, Guangdong Provincial Key Laboratory of Solid Wastes Pollution Control and Recycling, South China University of Technology, Guangzhou, Guangdong 510006, P. R. China

* Guangdong Engineering and Technology Research Center for Environmental Nanomaterials, South China University of Technology, Guangzhou, Guangdong 510006, P. R. China

$\S$ Jiangsu Academy of Science and Technology for Development, Nanjing, Jiangsu 210042, P. R. China

// School of Metallurgy and Environment, Central South University, Changsha 410083, P. R. China

Correspondence and requests for materials should be addressed to Zhang Lin. (Email: zlin@scut.edu.cn)

Content

S1. Supplemental Materials and Experiments

S2. Supplemental Results and Discussions

14 pages, 10 figures, 7 tables, and 5 equations 


\section{S1. Supplemental Materials and Experiments}

\section{Synthesis of brownmillerite and larnite in simulated COPR}

For the preparation of simulated COPR, two phases which do not host $\mathrm{Cr}(\mathrm{VI})$ were prepared by the methods below.

The brownmillerite compound was prepared by standard solid state reaction method. $\mathrm{CaCO}_{3}, \mathrm{Fe}_{2} \mathrm{O}_{3}$ and $\mathrm{Al}_{2} \mathrm{O}_{3}$ (99.99\% purity) were uniformly grinded and mixed. Then the precursors were sintered at $1300^{\circ} \mathrm{C}$ for $4 \mathrm{~h}$.

The larnite was synthesized using $\mathrm{CaO}$ and $\mathrm{SiO}_{2}$. After preconditioning the pellets of the mixture of starting material were sintered at $1140^{\circ} \mathrm{C}$ for $2 \mathrm{~h}$, the product was put into air for quenching when the temperature dropped down to $350^{\circ} \mathrm{C}$. The sample was grinded for $\mathrm{Cr}(\mathrm{VI})$ capture experiments.

Hydrotalcite with chloride in the interlayer was synthesized by co-precipitation method. The mixed aqueous solutions $(50 \mathrm{~mL})$ of $\mathrm{MgCl}_{2} \cdot 6 \mathrm{H}_{2} \mathrm{O}(0.8 \mathrm{~mol} / \mathrm{L})$ and $\mathrm{AlCl}_{3} \cdot 6 \mathrm{H}_{2} \mathrm{O}(0.4 \mathrm{~mol} / \mathrm{L})$ salts were co-precipitated with $50 \mathrm{~mL}$ of $6 \mathrm{~mol} / \mathrm{L}$ aqueous solution of $\mathrm{NaOH}$. During the process, the temperature was maintained at room temperature and $\mathrm{pH}$ was greater than 10 . The addition was completed within $3-4 \mathrm{~h}$. And the suspension was aged for $24 \mathrm{~h}$ at room temperature. The product was centrifuged and washed with deionized water for 3-4 times. Finally, the solid was freeze dried to give $\mathrm{Mg}-\mathrm{Al}-\mathrm{LDH}$ as white powder.

\section{Analyses of the $\mathrm{Cr}(\mathrm{VI})$ uptake data}

The capture capacity of $\mathrm{Cr}(\mathrm{VI})$ by different mineral phases was calculated with the following equation:

$$
q_{e}=\frac{\left(C_{0}-C_{e}\right) \times V}{m}
$$

where,

$q_{e}: \mathrm{Cr}(\mathrm{VI})$ capture amount at equilibrium $(\mathrm{mg} / \mathrm{g})$

$C_{0}$ : initial concentration of $\mathrm{Cr}(\mathrm{VI})(\mathrm{mg} / \mathrm{L})$;

$C_{e}$ : equilibrium concentration of $\mathrm{Cr}(\mathrm{VI})(\mathrm{mg} / \mathrm{L})$;

$V$ : total solution volume (L);

$m$ : dosage of the mineral phase $(\mathrm{g} / \mathrm{L})$.

The distribution coefficient was calculated with the following equation:

$$
k_{d}=q_{e} / C_{e} \times 1000
$$

where,

$k_{d}$ : distribution coefficient of $\mathrm{Cr}(\mathrm{VI})$ on the mineral phase $(\mathrm{mL} / \mathrm{g})$;

$q_{e}: \mathrm{Cr}(\mathrm{VI})$ capture amount at equilibrium $(\mathrm{mg} / \mathrm{g})$;

$C_{e}$ : equilibrium concentration of $\mathrm{Cr}(\mathrm{VI})(\mathrm{mg} / \mathrm{L})$; 
Nonlinear Langmuir (Eq. S3) and Freundlich models (Eq. S4) were employed to fit the $\mathrm{Cr}(\mathrm{VI})$ capture isotherms of hydrocalumite, which were fitted with the following equations:

$$
\frac{C_{e}}{q_{e}}=\frac{C_{e}}{Q_{m}}+\frac{1}{Q_{m} K_{L}}
$$

where,

$C_{e}$ : equilibrium concentration of $\mathrm{Cr}(\mathrm{VI})(\mathrm{mg} / \mathrm{L})$;

$q_{e}: \mathrm{Cr}(\mathrm{VI})$ capture amount at equilibrium $(\mathrm{mg} / \mathrm{g})$;

$Q_{m}$ : maximum $\mathrm{Cr}(\mathrm{VI})$ capture capacity $(\mathrm{mg} / \mathrm{g})$;

$K_{L}:$ Langmuir affinity coefficient $(\mathrm{L} / \mathrm{mg})$.

$$
q_{e}=K_{f} C_{e}^{n}
$$

where,

$C_{e}$ : equilibrium concentration of $\mathrm{Cr}(\mathrm{VI})(\mathrm{mg} / \mathrm{L})$;

$q_{e}: \mathrm{Cr}(\mathrm{VI})$ capture amount at equilibrium $(\mathrm{mg} / \mathrm{g})$;

$K_{f}$ : Freundlich affinity coefficient $(\mathrm{mg} / \mathrm{g})$;

$n$ : Freundlich exponential coefficient.

The adsorption energy in DFT calculation was defined as:

$$
E_{\text {ads }}=E_{\text {total }}-E_{\text {slab }}-E_{\mathrm{A}}
$$

where $E_{\text {total }}$ is the total energies of hydrocalumite with a bonded $\operatorname{Cr}(\mathrm{VI}), \mathrm{E}_{\text {slab }}$ is the energy of hydrocalumite, $\mathrm{E}_{\mathrm{A}}$ is the energy of isolated $\mathrm{Cr}(\mathrm{VI})$. 
S2. Supplemental Results and Discussions
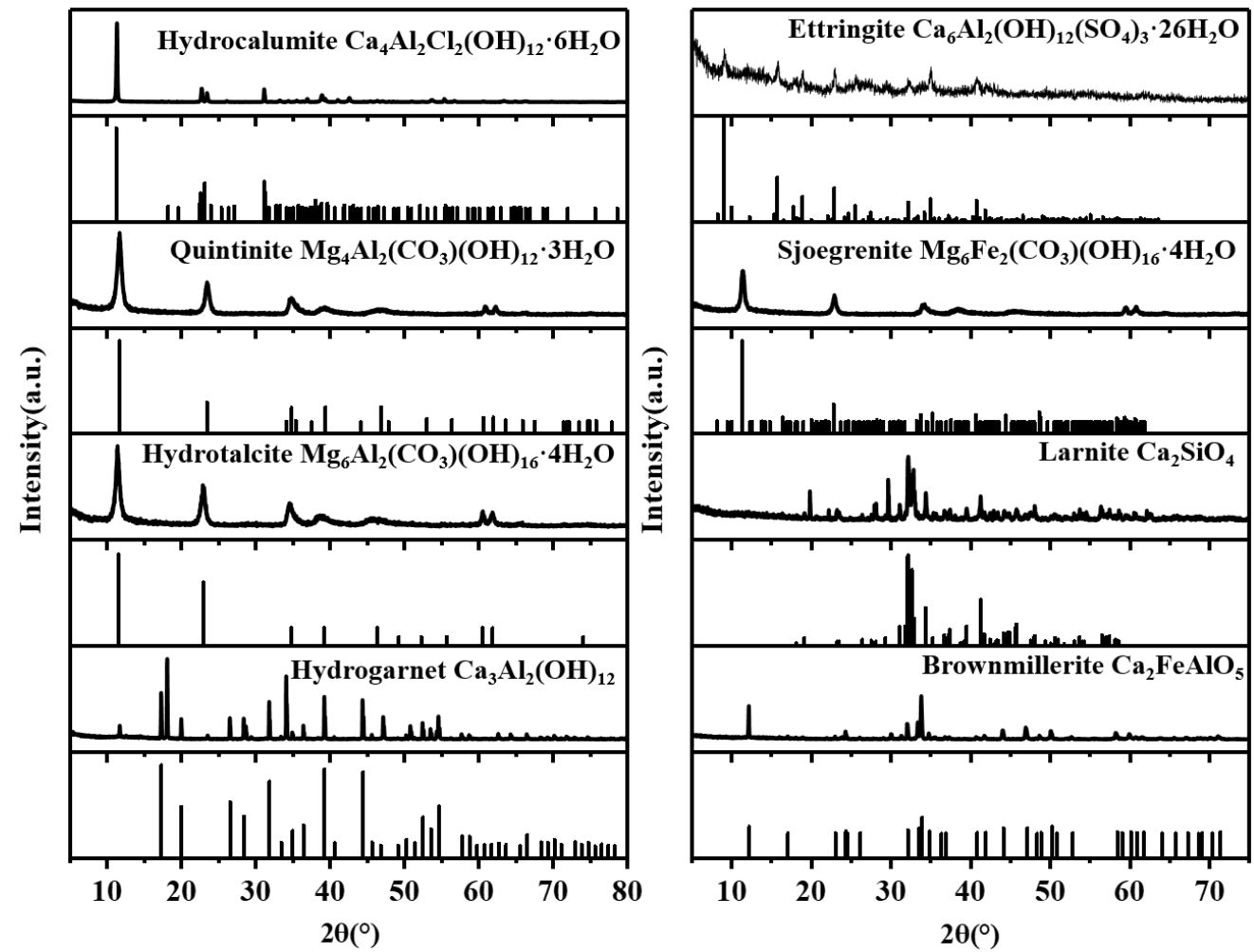

Figure S1. XRD patterns of prepared mineral phases in simulated COPR. 
Table S1. The chemical mineralogy and quantitative composition of O, COPR-H, and COPR-N

\begin{tabular}{|c|c|c|}
\hline & Chemical formula & $\%$ wt. \\
\hline \multicolumn{3}{|c|}{ Component $\mathrm{O}$ (phases without $\mathrm{Cr}(\mathrm{VI})$ existing) } \\
\hline brownmillerite & $\mathrm{Ca}_{2} \mathrm{FeAlO}_{5}$ & 51.0 \\
\hline brucite & $\mathrm{Mg}(\mathrm{OH})_{2}$ & 14.3 \\
\hline periclase & $\mathrm{MgO}$ & 8.2 \\
\hline pordlandite & $\mathrm{Ca}(\mathrm{OH})_{2}$ & 4.1 \\
\hline quartz & $\mathrm{SiO}_{2}$ & 6.1 \\
\hline calcite & $\mathrm{CaCO}_{3}$ & 14.3 \\
\hline larnite & $\mathrm{Ca}_{2} \mathrm{SiO}_{4}$ & 2.0 \\
\hline \multicolumn{3}{|c|}{ COPR-H } \\
\hline brownmillerite & $\mathrm{Ca}_{2} \mathrm{FeAlO}_{5}$ & 33.8 \\
\hline brucite & $\mathrm{Mg}(\mathrm{OH})_{2}$ & 9.5 \\
\hline periclase & $\mathrm{MgO}$ & 5.4 \\
\hline pordlandite & $\mathrm{Ca}(\mathrm{OH})_{2}$ & 2.7 \\
\hline quartz & $\mathrm{SiO}_{2}$ & 4.1 \\
\hline calcite & $\mathrm{CaCO}_{3}$ & 9.5 \\
\hline larnite & $\mathrm{Ca}_{2} \mathrm{SiO}_{4}$ & 1.4 \\
\hline hydrogarnet & $\mathrm{Ca}_{3} \mathrm{Al}_{2}(\mathrm{OH})_{12}$ & 18.9 \\
\hline hydrocalumite & $\mathrm{Ca}_{4} \mathrm{Al}_{2} \mathrm{Cl}_{2}(\mathrm{OH})_{12} \cdot 6 \mathrm{H}_{2} \mathrm{O}$ & 6.8 \\
\hline quintinite & $\mathrm{Mg}_{4} \mathrm{Al}_{2}\left(\mathrm{CO}_{3}\right)(\mathrm{OH})_{12} \cdot 3 \mathrm{H}_{2} \mathrm{O}$ & 5.4 \\
\hline sjoegrenite & $\mathrm{Fe}_{6} \mathrm{Al}_{2}\left(\mathrm{CO}_{3}\right)(\mathrm{OH})_{16} \cdot 4 \mathrm{H}_{2} \mathrm{O}$ & 1.4 \\
\hline ettringite & $\mathrm{Ca}_{6} \mathrm{Al}_{2}(\mathrm{OH})_{12}\left(\mathrm{SO}_{4}\right)_{3} \cdot 26 \mathrm{H}_{2} \mathrm{O}$ & 1.4 \\
\hline \multicolumn{3}{|c|}{ COPR-N } \\
\hline brownmillerite & $\mathrm{Ca}_{2} \mathrm{FeAlO}_{5}$ & 36.2 \\
\hline brucite & $\mathrm{Mg}(\mathrm{OH})_{2}$ & 10.1 \\
\hline periclase & $\mathrm{MgO}$ & 5.8 \\
\hline pordlandite & $\mathrm{Ca}(\mathrm{OH})_{2}$ & 2.9 \\
\hline quartz & $\mathrm{SiO}_{2}$ & 4.3 \\
\hline calcite & $\mathrm{CaCO}_{3}$ & 10.1 \\
\hline larnite & $\mathrm{Ca}_{2} \mathrm{SiO}_{4}$ & 1.4 \\
\hline hydrogarnet & $\mathrm{Ca}_{3} \mathrm{Al}_{2}(\mathrm{OH})_{12}$ & 20.3 \\
\hline quintinite & $\mathrm{Mg}_{4} \mathrm{Al}_{2}\left(\mathrm{CO}_{3}\right)(\mathrm{OH})_{12} \cdot 3 \mathrm{H}_{2} \mathrm{O}$ & 5.8 \\
\hline sjoegrenite & $\mathrm{Fe}_{6} \mathrm{Al}_{2}\left(\mathrm{CO}_{3}\right)(\mathrm{OH})_{16} \cdot 4 \mathrm{H}_{2} \mathrm{O}$ & 1.4 \\
\hline ettringite & $\mathrm{Ca}_{6} \mathrm{Al}_{2}(\mathrm{OH})_{12}\left(\mathrm{SO}_{4}\right)_{3} \cdot 26 \mathrm{H}_{2} \mathrm{O}$ & 1.4 \\
\hline
\end{tabular}

For every mixture group the mass ratio of brownmillerite: brucite: periclase: pordlandite: quartz: calcite: larnite: hydrogarnet: hydrocalumite: quintinite: sjoegrenite: ettringite was fixed to be $25: 7: 4: 2: 3: 7: 1: 14: 5: 4: 1: 1$. 
Table S2. Adsorption capacity of hydrocalumite mixed with other phases

\begin{tabular}{ccccc}
\hline Sample & COPR-5 & COPR-12 & COPR-25 & COPR-50 \\
\hline Mass ratio & $1: 20$ & $1: 7$ & $1: 3$ & $1: 1$ \\
(hydrocalumite:other phases) & & 7 & 13.13 & 19 \\
Uptake capacity $(\mathrm{mg} / \mathrm{g})$ & 2.25 & 87 & 355 & 790 \\
Binding affinity $\left(K_{\mathrm{d}}, \mathrm{mL} / \mathrm{g}\right)$ & 20 & &
\end{tabular}
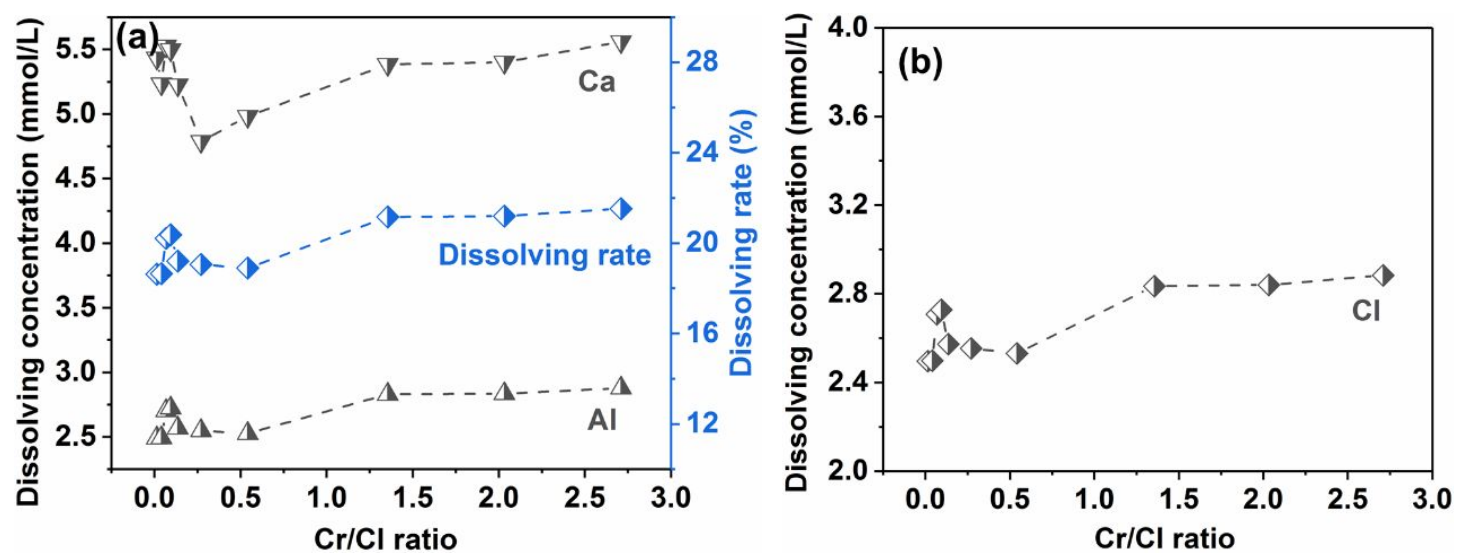

Figure S2. (a) Release of $\mathrm{Ca}^{2+}, \mathrm{Al}^{3+}$ and the corresponding dissolving rate of hydrocalumite. (b) Dissolving concentration of $\mathrm{Cl}^{-}$calculated from (a) with different $\mathrm{Cr} / \mathrm{Cl}$ ratios.

It can be seen that the dissolved mole ratios of $\mathrm{Ca} / \mathrm{Al}$ are around 1.9 2.2 (Figure $\mathrm{S} 2 \mathrm{a}$ ) under different $\mathrm{Cr} / \mathrm{Cl}$ ratios, which are very close to the stoichiometric $\mathrm{Ca} / \mathrm{Al}$ ratio of 2.0 in hydrocalumite $\left(\mathrm{Ca}_{4} \mathrm{Al}_{2} \mathrm{Cl}_{2}(\mathrm{OH})_{12} \cdot 6 \mathrm{H}_{2} \mathrm{O}\right)$. Therefore, according to the hydrocalumite concentration of $6.7 \mathrm{mmol} / \mathrm{L}$, it can be calculated from the dissolved $\mathrm{Ca}^{2+}$ and $\mathrm{Al}^{3+}$ that the dissolved amounts of hydrocalumite with different $\mathrm{Cr} / \mathrm{Cl}$ ratios were all around $\sim 20 \%$ (blue lines in Figure S2a). According to this value, the dissolving content of $\mathrm{Cl}$ with different $\mathrm{Cr} / \mathrm{Cl}$ ratios can be calculated, as shown in Figure $\mathrm{S} 2 \mathrm{~b}$. 


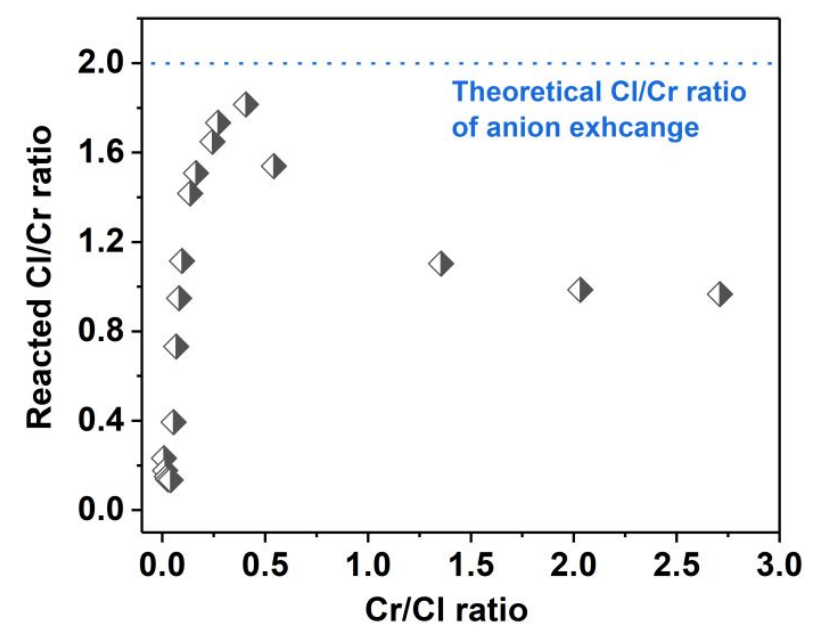

Figure S3. Reacted $\mathrm{Cl} / \mathrm{Cr}$ ratios through ion exchange between $\mathrm{Cr}(\mathrm{VI})$ and hydrocalumite. The dissolved $\mathrm{Cl}^{-}$was deducted according to Figure S2b.

Table S3. Structural parameters of Cr(VI)-loaded samples from $\mathrm{Cr} K$-edge EXAFS Data

\begin{tabular}{|c|c|c|c|c|c|}
\hline Sample & Atomic path & ${ }^{\mathrm{a}} \mathrm{CN}$ & ${ }^{b} \mathrm{R}(\AA)$ & ${ }^{c} \sigma^{2}(\AA)$ & ${ }^{\mathrm{d} R}$-factor \\
\hline A1 & $\mathrm{Cr}-\mathrm{O}^{e}$ & $3.7(4)$ & $1.64(2)$ & 0.002 & \multirow{2}{*}{0.014} \\
\hline$(\mathrm{Cr} / \mathrm{Cl}=0.014)$ & $\mathrm{Cr}-\mathrm{Ca} f$ & $1.7(5)$ & $4.06(2)$ & $0.009(3)$ & \\
\hline $\mathrm{A} 2$ & $\mathrm{Cr}-\mathrm{O}^{e}$ & $3.6(4)$ & $1.64(2)$ & 0.002 & \multirow{2}{*}{0.007} \\
\hline$(\mathrm{Cr} / \mathrm{Cl}=0.028)$ & $\mathrm{Cr}-\mathrm{Ca}^{f}$ & $1.8(2)$ & $3.93(6)$ & $0.008(6)$ & \\
\hline B1 & $\mathrm{Cr}-\mathrm{O}^{e}$ & $3.9(3)$ & $1.64(1)$ & 0.002 & \multirow{2}{*}{0.016} \\
\hline$(\mathrm{Cr} / \mathrm{Cl}=0.06)$ & $\mathrm{Cr}-\mathrm{Ca}^{f}$ & $1.8(5)$ & $3.88(3)$ & 0.005 & \\
\hline B2 & $\mathrm{Cr}-\mathrm{O}^{e}$ & $3.6(6)$ & $1.64(1)$ & $0.004(3)$ & \multirow{2}{*}{0.007} \\
\hline$(\mathrm{Cr} / \mathrm{Cl}=0.08)$ & $\mathrm{Cr}-\mathrm{Ca}{ }^{f}$ & $1.7(8)$ & $3.86(3)$ & $0.006(4)$ & \\
\hline $\mathrm{B} 3(\mathrm{Cr} / \mathrm{Cl}=0.14)$ & $\mathrm{Cr}-\mathrm{O}^{g}$ & $3.9(7)$ & $1.66(1)$ & 0.002 & 0.019 \\
\hline $\mathrm{B} 4(\mathrm{Cr} / \mathrm{Cl}=0.28)$ & $\mathrm{Cr}-\mathrm{O}^{g}$ & $3.3(2)$ & $1.67(1)$ & 0.002 & 0.014 \\
\hline $\mathrm{C} 1(\mathrm{Cr} / \mathrm{Cl}=1.4)$ & $\mathrm{Cr}-\mathrm{O}$ & $2.5(3)$ & $1.66(1)$ & 0.002 & 0.014 \\
\hline $\mathrm{C} 2(\mathrm{Cr} / \mathrm{Cl}=2.8)$ & $\mathrm{Cr}-\mathrm{O}$ & $2.1(3)$ & $1.66(2)$ & 0.002 & 0.019 \\
\hline
\end{tabular}

${ }^{\mathrm{a}} \mathrm{CN}$ : number of neighbors. ${ }^{\mathrm{b}} \mathrm{R}(\AA)$ : Interatomic distances. ${ }^{\mathrm{c}} \sigma^{2}(\AA)$ : Debye-Waller factor. ${ }^{\mathrm{d}} \mathrm{R}-$ factor: goodness of fitting. $\mathrm{Cr}-\mathrm{O}^{e}$ and $\mathrm{Cr}-\mathrm{Ca}^{f}$ were fitted with $\mathrm{CaCrO}_{4} \cdot\left(\mathrm{H}_{2} \mathrm{O}\right)_{2}$ reference, and $\mathrm{Cr}-\mathrm{O}^{g}$ was fitted with $\mathrm{Na}_{2} \mathrm{CrO}_{4}$ reference. The amplitude reduction factor $\left(\mathrm{S}^{2}\right)$ was fixed of 0.95 . Numbers in the parentheses are standard errors of the fitted parameters.

It can be calculated from Figure S3 Ca content that participated in $\mathrm{Cr}$-Ca coordination at $\mathrm{B} 2$ was only $1.7 \%$ of its total content in hydrocalumite, with the form of $\mathrm{Ca}-\mathrm{O}-\mathrm{Cr}$ $\mathrm{O}_{3}$ complex (Figure 2e, $2 \mathrm{f}$ and Table $\mathrm{S} 3$ ). Such low proportion suggested that $\mathrm{Cr}-\mathrm{Ca}$ coordination could not be realized by the competition of $\mathrm{Cr}$ with the original coordination structure of $\mathrm{Ca}$ on the layer $\left(\mathrm{Ca}_{4} \mathrm{Al}_{2}(\mathrm{OH})_{12}\right)$, but more likely due to the generation of partial undersaturated $\mathrm{Ca}$ sites, which would further form strong active 
sites for $\mathrm{Cr}(\mathrm{VI})$ coordination. This result was in accordance with Bing's work, ${ }^{5}$ which reported that $\mathrm{Ca}-\mathrm{Al} \mathrm{LDH}$ was more likely to form a distorted $\mathrm{Ca}(\mathrm{OH})_{6}$ octahedron with an additional $\mathrm{Ca}-\mathrm{OH}$ groups that provides more active coordination sites. However, under the premise of ensuring the integrity of hydrocalumite, the content of undersaturated sites could not be high, thus reach saturation easily. 


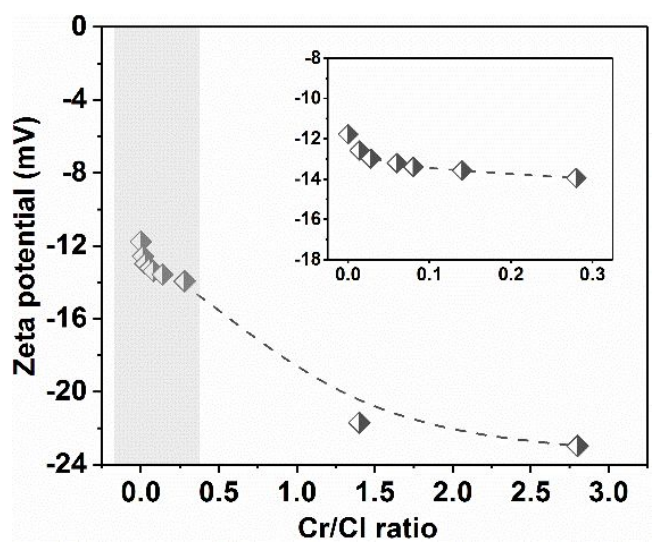

Figure S4. Zeta potentials of $\mathrm{Cr}(\mathrm{VI})$-bound hydrocalumite with different $\mathrm{Cr} / \mathrm{Cl}$ ratios

Table S4. Adsorption energy $\left(E_{\mathrm{ad}}\right)$ of different LDHs for targeted substances.

\begin{tabular}{|c|c|c|c|c|}
\hline LDHs & $\begin{array}{c}\text { Modification } \\
\text { method }\end{array}$ & $\begin{array}{r}\text { Targeted } \\
\text { substance }\end{array}$ & $\begin{array}{l}\text { Adsorption } \\
\text { energy }\left(E_{\mathrm{ad}}\right)\end{array}$ & Reference \\
\hline $\mathrm{Mg} / \mathrm{Al}-\mathrm{OH}$ & Exfoliation & Alcohol & -0.82 & 1 \\
\hline $\mathrm{Co} / \mathrm{Al}-\mathrm{OH}$ & Exfoliation & Benzyl alcohol & -1.5 & 2 \\
\hline $\mathrm{Ni} / \mathrm{Fe}-\mathrm{CO}_{3}$ & Exfoliation & Gold & -5.6 & 3 \\
\hline $\mathrm{Ni} / \mathrm{Fe}-\mathrm{Cl}$ & Cobalt intercalation & Water & -4.9 & 4 \\
\hline $\mathrm{Ca} / \mathrm{Al}-\mathrm{NO}_{3}$ & $\begin{array}{l}\text { Calcination and } \\
\text { rehydration }\end{array}$ & Isobutyraldehyde & -0.32 & 5 \\
\hline $\mathrm{Mg} / \mathrm{Al}-\mathrm{NO}_{3}$ & $\begin{array}{l}\text { Calcination and } \\
\text { rehydration }\end{array}$ & Isobutyraldehyde & -0.62 & 5 \\
\hline $\mathrm{Ca} / \mathrm{Al}-\mathrm{CO}_{3}$ & $\begin{array}{c}\text { Glycerol- } \\
\text { modification }\end{array}$ & Uranium & -4.0 & 6 \\
\hline $\mathrm{Ni} / \mathrm{Al}-\mathrm{CO}_{3}$ & $\begin{array}{c}\text { Glycerol- } \\
\text { modification }\end{array}$ & Uranium & -3.1 & 6 \\
\hline $\mathrm{Ca} / \mathrm{Al}-\mathrm{Cl}$ & - & Chromium & -4.7 & This work \\
\hline
\end{tabular}

Table S5. Isotherm Parameters of Cr(VI) capture by hydrocalumite.

\begin{tabular}{ccccccc}
\hline & \multicolumn{3}{c}{ Langmuir constants } & \multicolumn{3}{c}{ Freundlich constants } \\
Cr(VI) & $\begin{array}{c}q_{\mathrm{m}} \\
(\mathrm{mg} / \mathrm{g})\end{array}$ & $\begin{array}{c}K_{\mathrm{L}} \\
(\mathrm{L} / \mathrm{mg})\end{array}$ & $\mathrm{R}^{2}$ & $\begin{array}{c}K_{\mathrm{F}} \\
(\mathrm{mg} / \mathrm{g})\end{array}$ & $\mathrm{n}$ & $\mathrm{R}^{2}$ \\
\hline Whole & 73.5 & 0.268 & 0.998 & 13.3 & 2.96 & 0.653 \\
Stage A & 120 & 0.285 & 0.922 & 18.7 & 1.12 & 0.992 \\
Stage C & 20.8 & 13.1 & 0.999 & 27.5 & 3.33 & 0.956 \\
Stage D & 74.6 & 0.0756 & 0.997 & 28.0 & 6.46 & 0.991 \\
\hline
\end{tabular}


Table S6. Structural parameters of $\mathrm{Cr}(\mathrm{VI})$-loaded hydrocalumite from $\mathrm{Cr} \mathrm{K}$-edge EXAFS Data of Figure $7 \mathrm{a}$ at different contacting times,.

\begin{tabular}{|c|c|c|c|c|c|}
\hline Sample & Atomic path & ${ }^{\mathrm{a}} \mathrm{CN}$ & ${ }^{b} \mathrm{R}(\AA)$ & ${ }^{c} \sigma^{2}(\AA)$ & ${ }^{\mathrm{d}} \mathrm{R}$-factor \\
\hline \multirow[t]{2}{*}{$\mathrm{Cr}-1$} & $\mathrm{Cr}-\mathrm{O}^{e}$ & $3.5(1)$ & $1.64(4)$ & 0.002 (4) & \multirow{2}{*}{0.008} \\
\hline & $\mathrm{Cr}-\mathrm{Ca}^{f}$ & $0.9(2)$ & $3.90(7)$ & $0.008(9)$ & \\
\hline \multirow[t]{2}{*}{$\mathrm{Cr}-5$} & $\mathrm{Cr}-\mathrm{O}^{g}$ & $3.8(3)$ & $1.66(2)$ & 0.003 & 0.012 \\
\hline & $\mathrm{Cr}-\mathrm{Ca}^{f}$ & $1.5(5)$ & $3.86(4)$ & 0.005 & 0.010 \\
\hline Cr-60 & $\mathrm{Cr}-\mathrm{O}^{g}$ & $3.3(3)$ & $1.66(2)$ & 0.003 & 0.015 \\
\hline
\end{tabular}

${ }^{\mathrm{a}} \mathrm{CN}$ : number of neighbors. ${ }^{\mathrm{b}} \mathrm{R}(\AA)$ : Interatomic distances. ${ }^{\mathrm{c}} \sigma^{2}(\AA)$ : Debye-Waller factor. ${ }^{\mathrm{d}} \mathrm{R}-$ factor: goodness of fitting. $\mathrm{Cr}-\mathrm{O}^{e}$ and $\mathrm{Cr}-\mathrm{Ca}^{f}$ were fitted with $\mathrm{CaCrO}_{4} \cdot\left(\mathrm{H}_{2} \mathrm{O}\right)_{2}$ reference, and $\mathrm{Cr}-\mathrm{O}^{g}$ was fitted with $\mathrm{Na}_{2} \mathrm{CrO}_{4}$ reference. The amplitude reduction factor $\left(\mathrm{S}^{2}\right)$ was fixed of 0.95 . Numbers in the parentheses are standard errors of the fitted parameters.

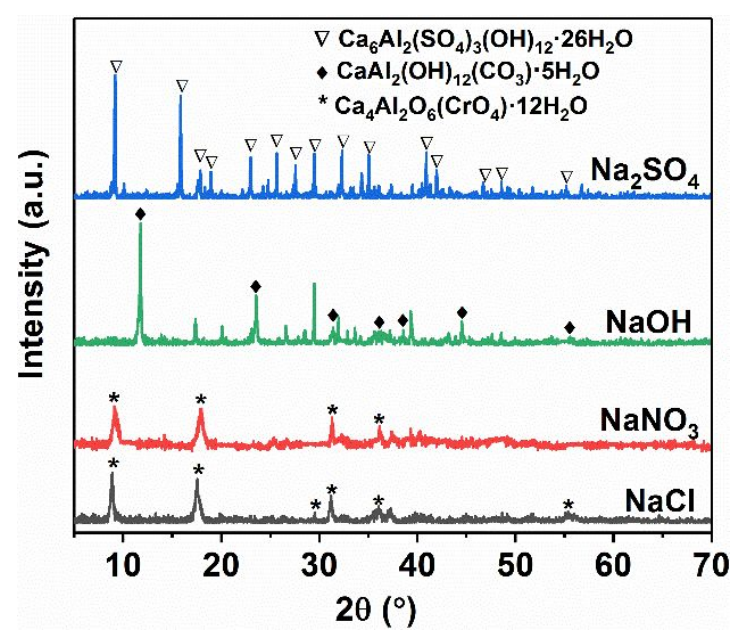

Figure S5. XRD patterns of $\mathrm{Cr}(\mathrm{VI})$-bound after eluted by $\mathrm{NaCl}, \mathrm{NaNO}_{3}, \mathrm{Na}_{2} \mathrm{SO}_{4}$, and $\mathrm{NaOH}$ solutions. 

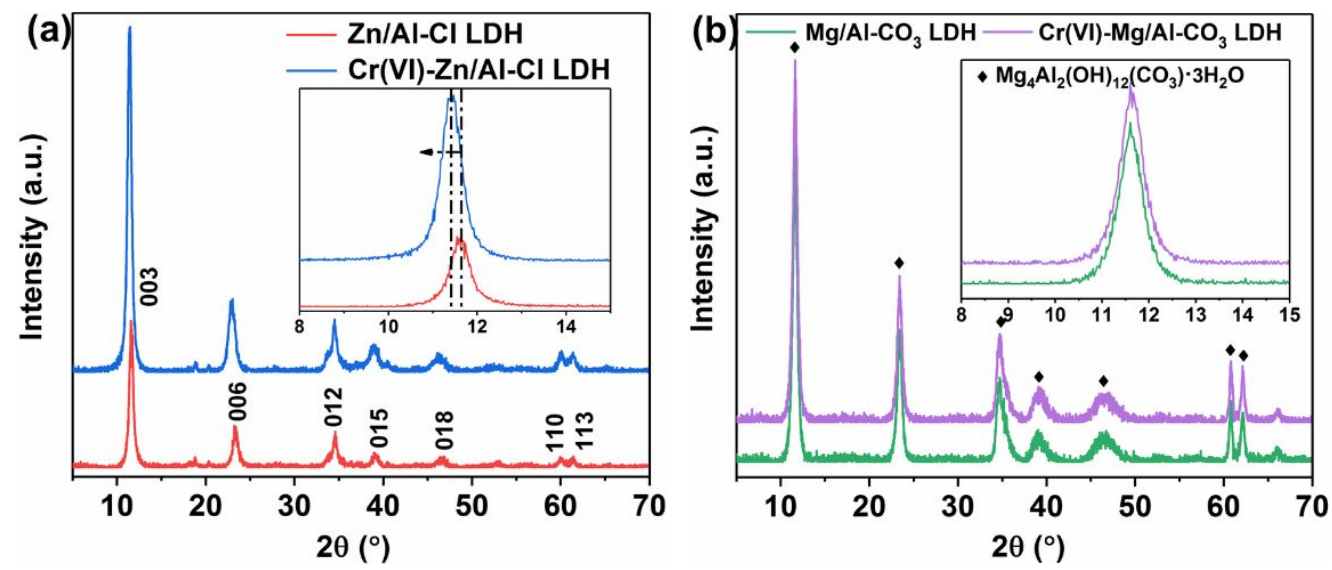

Figure S6. XRD patterns of $\mathrm{Zn} / \mathrm{Al}-\mathrm{Cl}$ and $\mathrm{Mg} / \mathrm{Al}-\mathrm{CO}_{3} \mathrm{LDHs}^{7}$ before and after $\mathrm{Cr}(\mathrm{VI})$ capture. The $\mathrm{Cr}(\mathrm{VI})$ initial concentration is $500 \mathrm{mg} / \mathrm{L}$.

It can be seen from Figure S4a that after $\mathrm{Cr}(\mathrm{VI})$ capture, a slight peak shift towards lower angle region was observed at in XRD pattern of $\mathrm{Zn} / \mathrm{Al}-\mathrm{Cl} \mathrm{LDH}$, which could be attributed to the increase of layer-to-layer distance. This increase suggested that $\mathrm{CrO}_{4}{ }^{2-}$ has entered into the gallery of $\mathrm{Zn} / \mathrm{Al}-\mathrm{Cl} \mathrm{LDH}$ by ion exchange with $\mathrm{Cl}^{-}$. However, no obvious of peak shift was observed in the XRD pattern of $\mathrm{Mg} / \mathrm{Al}-\mathrm{CO}_{3} \mathrm{LDH}$ after $\mathrm{Cr}(\mathrm{VI})$ capture, suggesting that no ion exchange occurred between $\mathrm{CrO}_{4}{ }^{2-}$ and $\mathrm{CO}_{3}{ }^{2-}$ during the capture process.
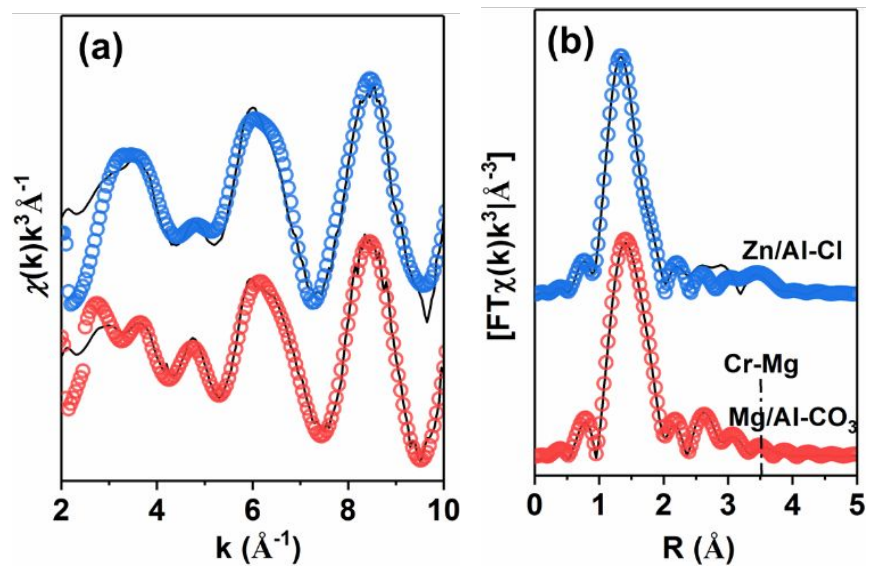

Figure S7. Normalized $k^{3}$-weighted experimental (lines) and their fitted (dots) $\mathrm{Cr} K$ edge EXAFS spectra of $\mathrm{Cr}(\mathrm{VI})$ capture by $\mathrm{Mg} / \mathrm{Al}-\mathrm{CO}_{3}$ and $\mathrm{Zn} / \mathrm{Al}-\mathrm{Cl} \mathrm{LDHs}$ (a), and the corresponding Fourier transformed magnitude (b). Data in red and blue color were fitted with $\mathrm{MgCrO}_{4}$ and $\mathrm{Na}_{2} \mathrm{CrO}_{4}$ references, respectively. The corresponding fitting data are listed in Table S7. 
Table S7. Structural parameters of $\mathrm{Cr}(\mathrm{VI})$-loaded Samples from $\mathrm{Cr} K$-edge EXAFS Data of Figure S7.

\begin{tabular}{cccccc}
\hline Sample & Atomic path & ${ }^{\mathrm{a}} \mathrm{CN}$ & ${ }^{\mathrm{b}} \mathrm{R}(\AA)$ & ${ }^{\mathrm{c}} \sigma^{2}(\AA)$ & ${ }^{\mathrm{d}} \mathrm{R}$-factor \\
\hline (A) & $\mathrm{Cr}-\mathrm{O}^{e}$ & $2.6(9)$ & $1.58(9)$ & 0.007 & \multirow{2}{*}{0.008} \\
& $\mathrm{Cr}-\mathrm{Mg}^{f}$ & $1.4(4)$ & $3.56(8)$ & $0.010(4)$ & \\
(B) & $\mathrm{Cr}-\mathrm{O}^{g}$ & $1.5(3)$ & $1.64(2)$ & $0.002(5)$ & 0.023
\end{tabular}

${ }^{\mathrm{a}} \mathrm{CN}$ : number of neighbors. ${ }^{\mathrm{b}} \mathrm{R}(\AA)$ : Interatomic distances. ${ }^{\mathrm{c}} \sigma^{2}(\AA)$ : Debye-Waller factor. ${ }^{\mathrm{d}} \mathrm{R}-$ factor: goodness of fitting. The amplitude reduction factor $\left(\mathrm{S}^{2}\right)$ was fixed of 0.95 . Numbers in the parentheses are standard errors of the fitted parameters.

$\mathrm{Cr}-\mathrm{Mg}$ second-neighbor contributions could be obviously observed in the R-space spectra of $\mathrm{Mg} / \mathrm{Al}-\mathrm{CO}_{3}$, which was suggestive for the surface coordination occurring between $\mathrm{Cr}(\mathrm{VI})$ and the layer of $\mathrm{Mg} / \mathrm{Al}-\mathrm{CO}_{3}$. However, the EXAFS spectra of $\mathrm{Zn} / \mathrm{Al}$ $\mathrm{Cl}$ revealed a better fit with the $\mathrm{Na}_{2} \mathrm{CrO}_{4}$ reference (Table S7), suggesting no surface coordination occurred during $\mathrm{Cr}(\mathrm{VI})$ capture. Since the XRD pattern of $\mathrm{Zn} / \mathrm{Al}-\mathrm{Cl}$ showed an obvious peak shift at $2 \theta=11.353^{\circ}$ towards lower angle region after $\mathrm{Cr}(\mathrm{VI})$ capture (Figure S6), interlayer ion exchange was supposed to be the predominated force for $\mathrm{Cr}(\mathrm{VI})$ binding by $\mathrm{Zn} / \mathrm{Al}-\mathrm{Cl} \mathrm{LDH}$. Moreover, no obvious peak transformation was observed in the XRD pattern of $\mathrm{Mg} / \mathrm{Al}-\mathrm{CO}_{3}$ after $\mathrm{Cr}(\mathrm{VI})$ binding, suggesting that no $\mathrm{Cr}(\mathrm{VI})$ anions intercalated into the galley and the $\mathrm{Cr}-\mathrm{Mg}$ coordination occurred on the outer layer. 

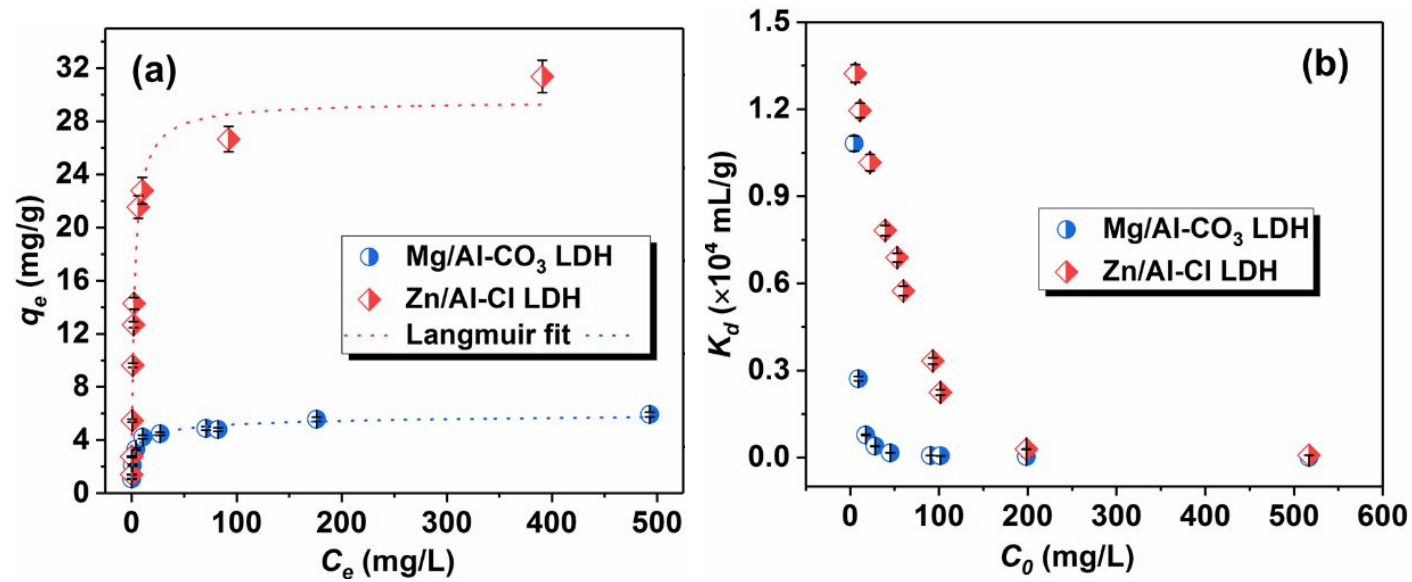

Figure S8. $\mathrm{Cr}(\mathrm{VI})$ capture isotherm and the corresponding $K_{\mathrm{d}}$ values by $\mathrm{Mg} / \mathrm{Al}-\mathrm{CO}_{3}$ and $\mathrm{Zn} / \mathrm{Al}-\mathrm{Cl}$ LDHs. The $\mathrm{LDH}$ dosages are $4.0 \mathrm{~g} / \mathrm{L}$, and the $\mathrm{Cr}(\mathrm{VI})$ initial concentrations are $5-500 \mathrm{mg} / \mathrm{L}$.

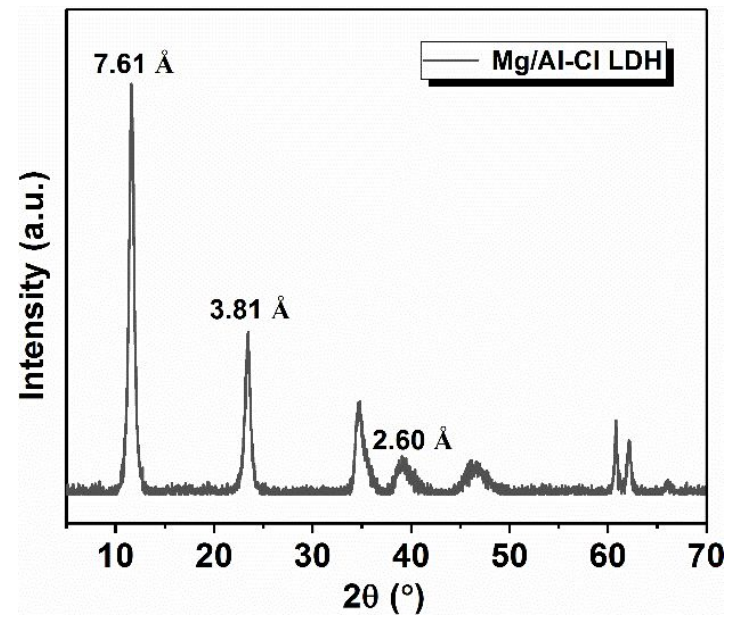

Figure S9. XRD pattern of $\mathrm{Mg} / \mathrm{Al}-\mathrm{Cl} \mathrm{LDH}^{8}$. 

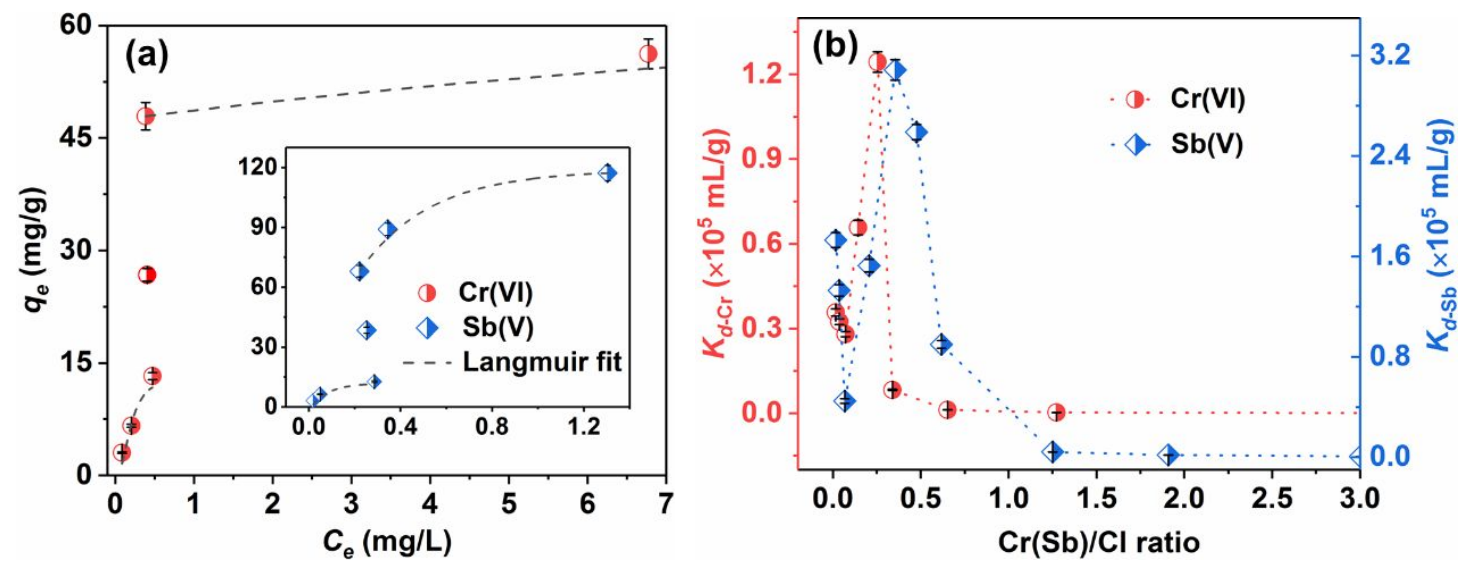

Figure S10. Capture isotherm of $\mathrm{SbO}_{4}{ }^{3-}$ and $\mathrm{CrO}_{4}{ }^{2}$ by $\mathrm{Mg} / \mathrm{Al}-\mathrm{Cl} \mathrm{LDH}$ (a) and the corresponding $k_{\mathrm{d}}$ values versus $\mathrm{Cr}(\mathrm{Sb}) / \mathrm{Cl}$ ratios (b). The $\mathrm{Mg} / \mathrm{Al}-\mathrm{Cl} \mathrm{LDH}$ dosage was $0.8 \mathrm{~g} / \mathrm{L}$. 


\section{References}

(1) Cuautli, C.; Valente, J. S.; Conesa, J. C.; Ganduglia-Pirovano, M. V.; Ireta, J. Theoretical Study of the Catalytic Performance of Activated Layered Double Hydroxides in the Cyanoethylation of Alcohols. J. Phys. Chem. C 2019, 123, 8777-8784.

(2) Wang, Q.; Chen, L.; Guan, S.; Zhang, X.; Wang, B.; Cao, X.; Yu, Z.; He, Y.; Evans, D. G.; Feng, J.; Li, D. Ultrathin and Vacancy-Rich Coal-Layered Double Hydroxide/Graphite Oxide Catalysts: Promotional Effect of Cobalt Vacancies and Oxygen Vacancies in Alcohol Oxidation. Acs Catal. 2018, 8, 3104-3115.

(3) Zhang, J.; Liu, J.; Xi, L.; Yu, Y.; Chen, N.; Sun, S.; Wang, W.; Lange, K. M.; Zhang, B. Single-Atom $\mathrm{Au} /$ Nife Layered Double Hydroxide Electrocatalyst: Probing the Origin of Activity for Oxygen Evolution Reaction. J. Am. Chem. Soc. 2018, 140, 3876-3879.

(4) Thenuwara, A. C.; Attanayake, N. H.; Yu, J.; Perdew, J. P.; Elzinga, E. J.; Yan, Q.; Strongin, D. R. Cobalt Intercalated Layered NiFe Double Hydroxides for the Oxygen Evolution Reaction. J. Phys. Chem. $B$ 2018, 122, 847-854.

(5) Bing, W.; Zheng, L.; He, S.; Rao, D.; Xu, M.; Zheng, L.; Wang, B.; Wang, Y.; Wei, M. Insights On Active Sites of CaAl-Hydrotalcite as a High-Performance Solid Base Catalyst Toward Aldol Condensation. Acs Catal. 2018, 8, 656-664.

(6) Zou, Y.; Liu, Y.; Wang, X.; Sheng, G.; Wang, S.; Ai, Y.; Ji, Y.; Liu, Y.; Hayat, T.; Wang, X. GlycerolModified Binary Layered Double Hydroxide Nanocomposites for Uranium Immobilization via Extended X-Ray Absorption Fine Structure Technique and Density Functional Theory Calculation. Acs Sustain. Chem. Eng. 2017, 5, 3583-3595.

(7) Zhou, M.; Yan, L.; Ling, H.; Diao, Y.; Pang, X.; Wang, Y.; Gao, K. Design and Fabrication of Enhanced Corrosion Resistance Zn-Al Layered Double Hydroxides Films Based Anion-Exchange Mechanism on Magnesium Alloys. Appl. Surf. Sci. 2017, 404, 246-253.

(8) Grover, K.; Komarneni, S.; Katsuki, H. Uptake of Arsenite by Synthetic Layered Double Hydroxides. Water Res. 2009, 43, 3884-3890. 